\title{
Optimización en la gestión de información en negocios de la ciudad de Tarija basado en aplicaciones móviles "Business app"
}

\author{
Optimization in information management in business in the city of \\ Tarija based on mobile applications "Business app"
}

\author{
Franz Alvaro Medrano, franz.medrano@icloud.com \\ Universidad Autónoma Juan Misael Saracho, Bolivia
}

\section{RESUMEN}

La ciudad de Tarija se encuentra en una época de una gran apertura de nuevos negocios de productos y servicios, administrados por gerentes dispuestos a publicitar y difamar la información de sus funcionalidades, para esto se propone el proyecto que consiste en la optimización en la gestión de información en negocios de la ciudad de Tarija basado en aplicaciones móviles, en el cual busca informar y dar a conocer a los distintos clientes y personas acerca de las funcionalidades de las mismas del negocio e interactuando para obtener más información. Esta App permitirá tener un control de la información detallada, actualizada de los diferentes negocios en la ciudad de Tarija tales como creación de nuevas categorías de negocio, creación de nuevos negocios emergentes, almacenar descripción de sus productos y servicios, horarios de apertura-cierre, galería de imágenes, localización de los mismos. El consumo de la App supone un acercamiento directo, y una comunicación entre los diferentes negocios que buscan ofrecer una imagen digital ofertando sus funcionalidades y los usuarios que navegan mediante Smartphone navegando y buscando requerimientos informativos de su interés. El presente proyecto está compuesto por los siguientes: C1: desarrollo de una aplicación móvil nativa que gestione la información y contenido informativo de los distintos negocios. C2: socialización acerca de la funcionalidad de la app hacia los usuarios.

Palabras clave: Smartphone; App; negocios emergentes

The city of Tarija is at a time of a great opening of new businesses of products and services, managed by managers willing to publicize and defame the information of their functionalities, for this the project is proposed that consists of the optimization in the management of information on business in the city of Tarija based on mobile applications, in which it seeks to inform and inform the different clients and people about the functionalities of the business and interacting to obtain more information. This App will allow you to have a detailed, updated information control of the different businesses in the city of Tarija such as creation of new business categories, creation of new emerging businesses, store description of your products and services, opening-closing times, gallery of images, location of them. The consumption of the App is a direct approach, and a communication between the different businesses that seek to offer a digital image offering their functionalities and the users who browse through Smartphone browsing and looking for informative requirements of their interest. The present project is composed of the following: C1: development of a native mobile application that manages the information and informative content of the different businesses. C2: socialization about the functionality of the app towards users.

Key words: Smartphone; App; emerging business 


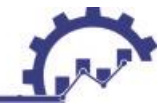

\section{INTRODUCCIÓN}

En los últimos años el crecimiento de la tecnología en los Smartphone ha generado grandes progresos en todos los ámbitos (comerciales, informativos, educación). Las comunicaciones fueron las de mayor desarrollo, especialmente el desarrollo en Internet. Esta influye en nuestras vidas y en nuestras costumbres, modificando la forma de buscar información, de entretenernos, de comunicarnos $\mathrm{y}$ por supuesto han aparecido nuevas formas de comprar y vender productos y servicios.

En la población de Tarija, Bolivia hoy en día las personas se comunican sin importar la distancia ya sea a través del Smartphone que ofrece Internet. Entre los cuales se encuentran los e-mails, mensajes de texto, foros públicos, redes sociales. Pero estos beneficios no solo satisfacen a las personas físicas (para nombrar a los consumidores finales), sino que las empresas o negocios también han encontrado grandes oportunidades en los desarrollos de las comunicaciones a través de un modelo de negocio de internet conocido como B2C1.

Partiendo de la premisa de que los costos de las comunicaciones $y$ propaganda se reducen y que estas tecnologías se encuentran al alcance tanto de grandes negocios, sucursales como así también de nuevos y pequeños. Cualquiera de estas puede estar al alcance de otras empresas o potenciales clientes dispersos alrededor de la ciudad.

A la vez las funcionalidades de las aplicaciones son diversas (como: Comunicación, Multimedia, Productividad, Entretenimiento, Juegos, etc.) y poco a poco están ingresando a nuestro mercado, aprovechando estas ventajas que son utilizadas en el día a día. Los nuevos negocios desean probar nuevas tecnologías mucho más renovadas $\mathrm{y}$ actualizadas para ofrecer calidad, información e interacción hacia sus clientes.

En la actualidad existen nuevas tecnologías en el área del desarrollo en la programación como ser SKD, librerías, API's de código abierto (liberados, por parte de las empresas Apple, Google, Facebook, bajo sus licencias respectivas), entonces se puede afirmar que se puede desarrollar nuevas Apps funcionales aprovechando a ser aplicadas en nuestro medio e innovarlo para facilitar $y$ automatizar las tareas diarias.

El mundo de las Apps se encuentra inmerso en la sociedad, y ahora en la ciudad de Tarija, está empezando a nacer, y se requiere que su población se actualice con los cambios actuales en relación al uso de la tecnología, logrando con ello, que esta se mantenga a la vanguardia de la era de la información y comunicación informado, con este estudio se procura un mundo más interactivo, automatizado e informativo.

Con el proyecto una vez implementado y puesto en producción, los usuarios lograron conectarse con la plataforma virtual a través de la aplicación móvil, logrando navegar y realizar distintas búsquedas según sus intereses, buscando en categorías, encontrando un negocio o sucursal en específico, además de un perfil informativo y una imagen del mismo, en donde los administradores y usuarios publican todo tipo de contenido informativo en servicios y productos, llegando a ofrecer una experiencia agradable de tal manera que pueda ser más visible, útil, óptimo para la búsqueda hacia los nuevos futuros clientes e incrementar sus ventas posteriores.

Para culminar es importante mencionar que la investigación tuvo propósito lograr la optimización en la gestión de información en negocios de la 
ciudad de Tarija basado en aplicaciones móviles "Business App" y así, satisfacer sus necesidades hacia la automatización de su información en la aplicación. Certificado por parte de los negocios avalando que la aplicación fue utilizada y aprobada. Se dispuso de la participación constante de los gerentes de negocios apoyando en la interactividad y funcionalidad del proyecto.

\section{MATERIALES Y MÉTODOS}

De acuerdo con el enfoque de los proyectos de aplicaciones móviles, se identifica con la siguiente metodología MADP (Mobile App Development Process) (Francis, 2017 y ICESI, 2009).) utilizada por el grupo "The BHW Group", la misma que es adaptada hacia este estudio y consta de las siguientes fases:

- Diseño de Experiencia del Usuario (UX).

- Diseño de Interfaz de Usuario (UI) y Diseño de Interacción (IxD).

- Diseño Técnico de Alto Nivel (Tech Stack).

- Revisión y Pruebas.

- Despliegue y Distribución de la App.

Es importante mencionar que este estudio contó con un proceso de socialización usando, material didáctico, manual de usuario de cómo usar la aplicación de negocio.

\section{RESULTADOS}

A continuación se describe el proceso de socialización de la App

\section{Socialización Acerca de la Funcionalidad de la App Hacia los Usuarios}

Con la implementación del proyecto se espera contar con una aplicación móvil inédita en el ámbito de la información optimizada y automatizada en una plataforma virtual que permitió a los negocios, clientes, usuarios un uso masivo de interacción mediante perfiles digitales, logrando soluciones de optimización de gestión de control informativo e interacción que actualmente las personas necesitan en el ámbito de un mejor servicio automatizado.

Beneficiaros: Administradores y Gerentes de Negocios, Clientes y usuarios

\section{Elaboración del Tutorial}

Se realizó la creación de un tutorial usando software llamado "Keynote" 1 creando pantallas de presentación e introducción de la aplicación para la socialización hacia la orientación e interacción de la misma hacia las personas voluntarias mediante el siguiente cronograma de actividades:

\section{Actividades:}

- Fase I. Obtención y descarga de la App de la página web

- Fase II. Uso y manejo de la funcionalidades de ña App

\section{Elaboración del Material Didáctico}

Se elaboró una serie de folletos que fueron entregados a los participantes, con imágenes ilustrativas y textos descriptivos, además de la página web que estuvo como presentación del mismo.

\section{Condiciones de Espacio de Trabajo}

Para la socialización se contó con un laboratorio de computadores dentro de la universidad, donde las personas lograron interactuar y realizar una recreación con el exponente. Las instalaciones del laboratorio conjuntamente con los equipos de computación fueron apropiadas para la discusió

\footnotetext{
${ }^{1}$ Keynote es una app de software de presentación desarrollada como parte del set de productividad iWork por Apple Inc.
} 


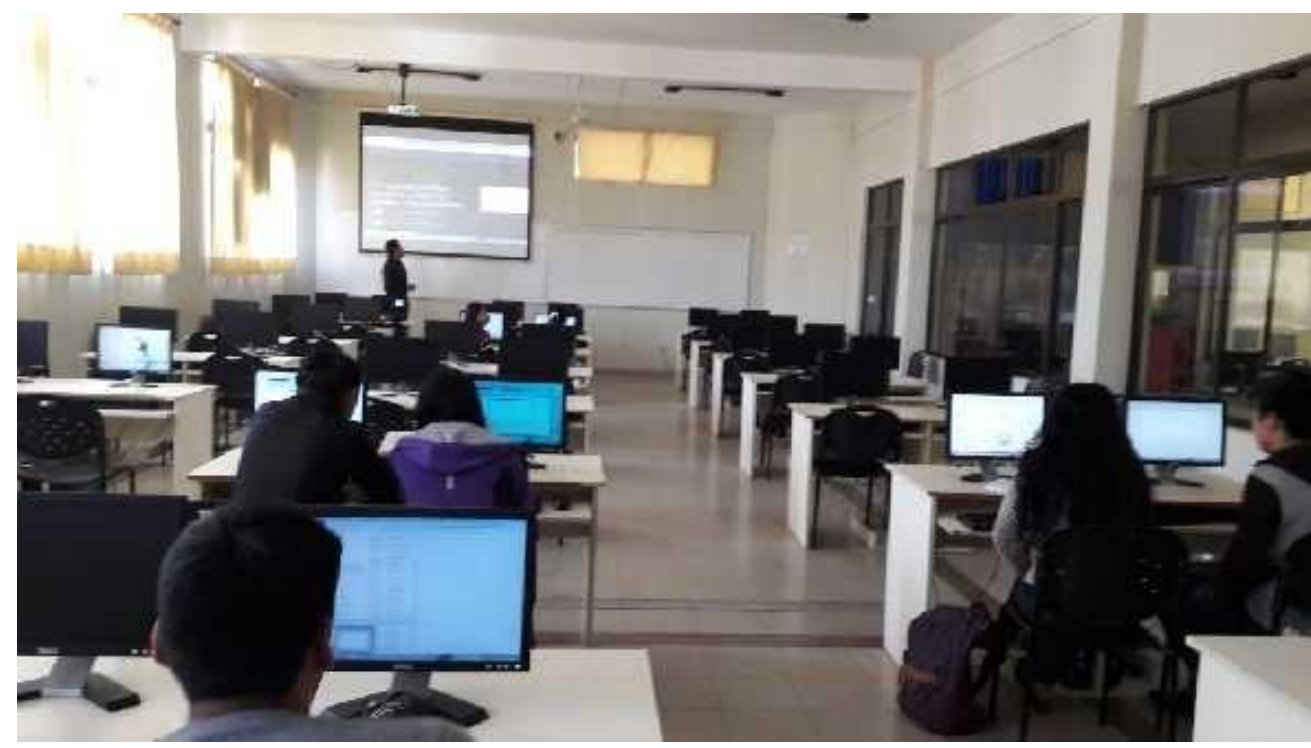

Figura 1. Explicación de la presentación de la página web a las personas

\section{Preparación de Lista de Asistencia}

La selección de los participantes fue valorada por la disposición mostrada por algunas personas que manifestaron su interés por formar parte en las actividades de socialización, para ello se realizó una preparación en la selección de los mismos, los cuales estuvieron dispuestas a apoyar con sus conocimientos y habilidades, en el cuadro 1 se describe los datos de los participante.

Cuadro 1. Datos de los participantes voluntarios de la socialización

\begin{tabular}{|c|c|c|c|}
\hline No & C.I. & NOMBRES & APELLIDOS \\
\hline 1 & 5807295 & José Luis & Zurita Lafuente \\
\hline 2 & 9018820 & Francisco José & Uriarte Salvador \\
\hline 3 & 9534314 & Carmen Julián & Castellanos Cortez \\
\hline 4 & 9024815 & Julio Fernando & Chávez Montero \\
\hline 5 & 3380384 & María Victoria & Popa Sales \\
\hline 6 & 6715511 & Elena María & Cristóbal Carrión \\
\hline 7 & 3647606 & Catalina Laura & De la Vega Lozano \\
\hline 8 & 7262553 & Víctor & Pacheco Pálala \\
\hline 9 & 1070956 & Cesar Manuel & Olmos Barbera \\
\hline 10 & 5896486 & Sebastian & Saavedra Callejo \\
\hline 11 & 6572857 & Cristina & Prieto Calle \\
\hline
\end{tabular}




\begin{tabular}{|c|c|c|c|}
\hline № & C.I. & NOMBRES & APELLIDOS \\
\hline 12 & 5629204 & Emilia & Pérez Roque \\
\hline 13 & 1078407 & Pablo Joel & Bustamante Romero \\
\hline 14 & 8130854 & Javier & Hoyos Arias \\
\hline 15 & 2614555 & Sergio Rodrigo & Serrano Fuentes \\
\hline 16 & 8650856 & Omar Alexis & García Santana \\
\hline 17 & 3913718 & Jorge & Cabrera Campos \\
\hline 18 & 3296447 & Andrea & Prieto Sánchez \\
\hline 19 & 2005184 & Julia & Rodríguez Gómez \\
\hline 20 & 4493130 & Diego Roberto & Morales Garcia \\
\hline 21 & 5242405 & Marcos & Ibáñez Hernández \\
\hline 22 & 5372204 & Rafael Mauricio & Alonso González \\
\hline 23 & 4282883 & Olivia & Fuentes Vidal \\
\hline 24 & 5718283 & Raquel & Rivera \\
\hline 25 & 4217108 & Iván Esteban & Méndez Serrano \\
\hline 26 & 3957073 & Teresa Ana & Navarro Moya \\
\hline 27 & 3658887 & Mateo David & Álvarez Ramos \\
\hline 28 & 6775450 & Samuel & Jiménez Cruz \\
\hline 29 & 3956987 & Enrique & Muñoz López \\
\hline 30 & 2191784 & Roció & Aguilar \\
\hline
\end{tabular}

\section{Preparación de Folletos del Proyecto}

Se procede a la creación folletos para la distribución y reparto a los usuarios voluntarios:
Elaboración de diapositivas y contenido de la capacitación

Las siguientes imágenes muestran el desarrollo y exposición de las diapositivas hacia nuestros usuarios. 


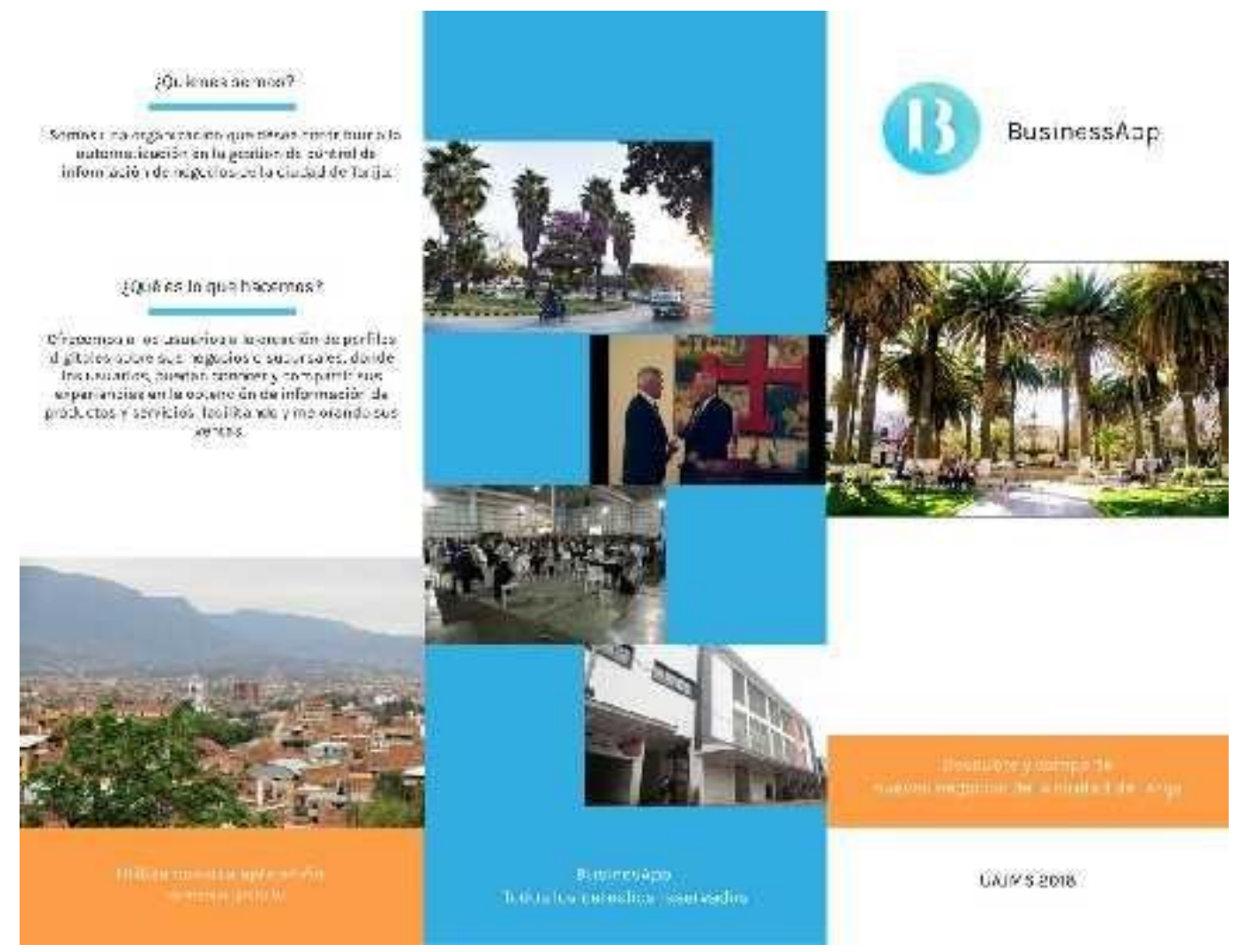

Figura 2. Presentación del folleto de la aplicación

\section{OPTIMIZACIÓN EN LA GESTIÓN DE INFORMACION EN NEGOCIOS DE LA CIUDAD DE TARIJA BASADO EN APLICACIONES MÓVILES BUSINESSAPP}

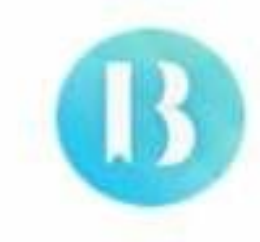

\section{Por: Franz Alvaro Medrano Duran}

Figura 3. Diapositivas de exposición 


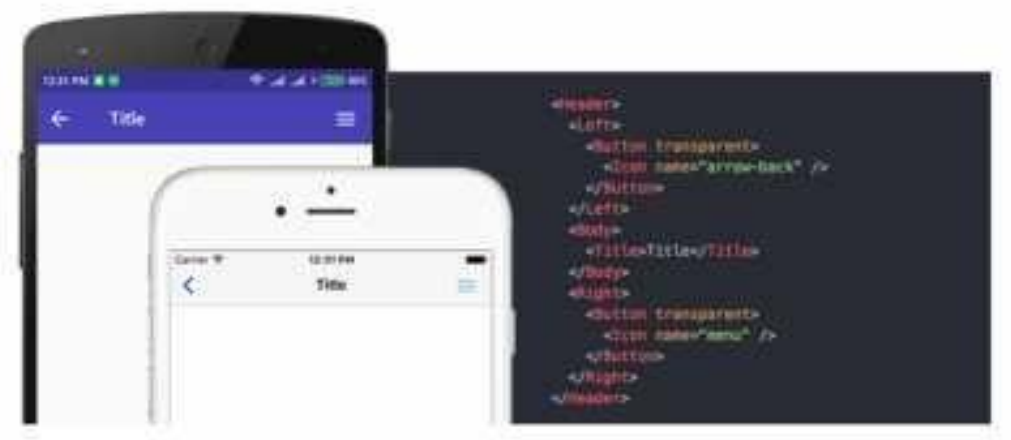

- Al desarroliar una app en Resct-Native, el codigo que escribimos es el mismo para las versiones de Android el lOS Solo tenemós quie preocuparnos de la logica del negocio y de maquetas una unica vez."

Figura 4. Concepto del lenguaje de programación
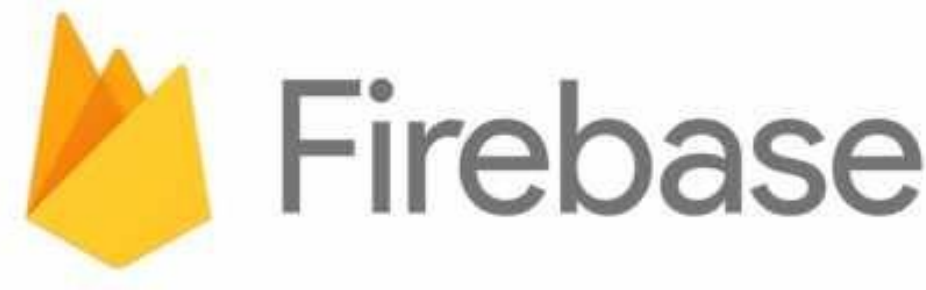

\section{Es una base de datos que accede a un servicio web con una API para guardar y sincronizar datos en la nube en tiempo real.}

Figura 5. Firebase un servicio de back-end 


\section{CARACTERISTICAS DE BASE DE DATOS FIREBASE}

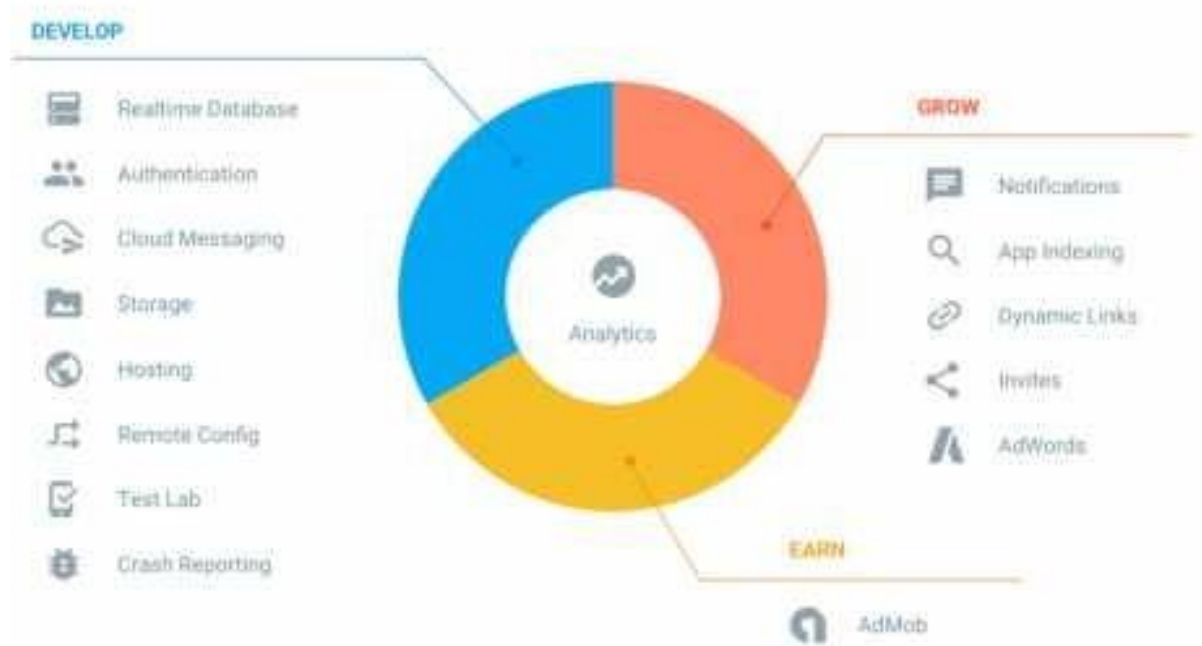

Figura 6. Características principales de firebase que utilizamos

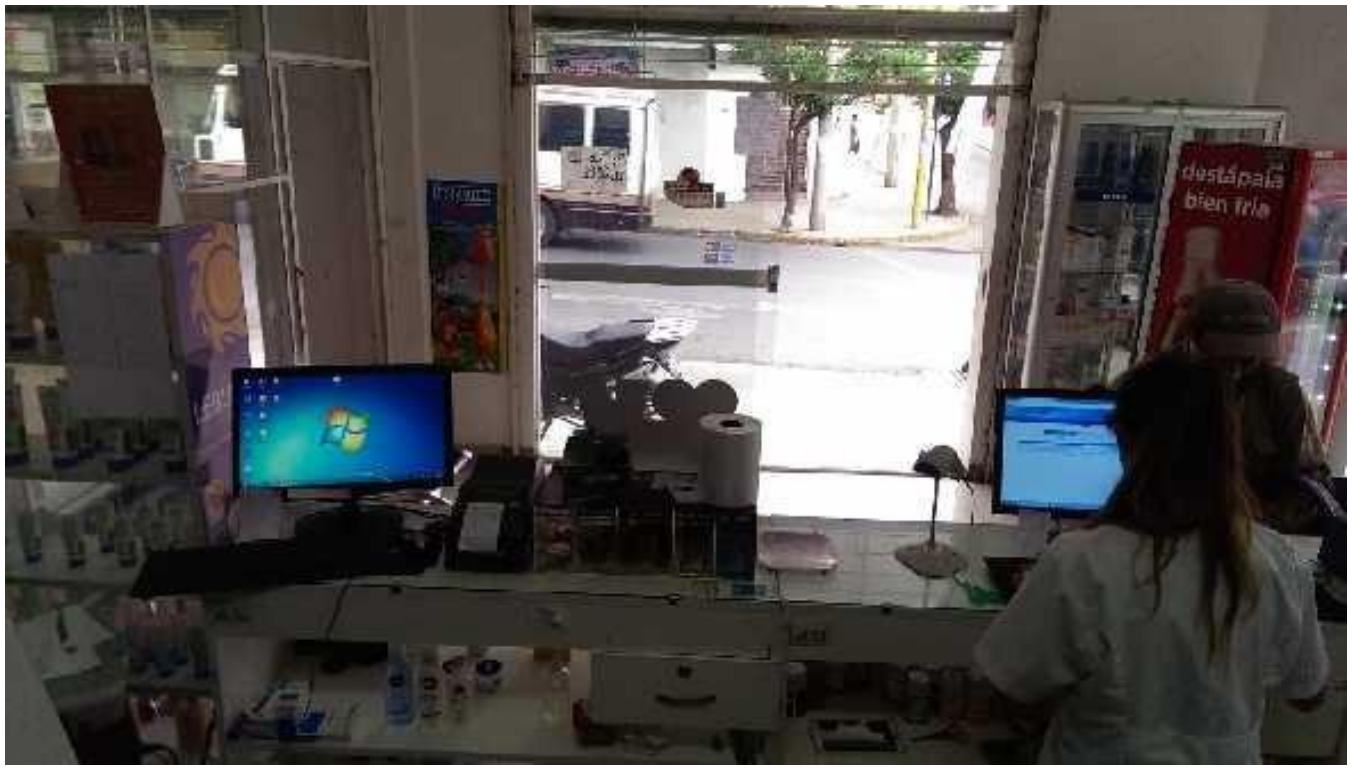

Figura 7. Fotografías de los negocios donde utilizo la aplicación №1 


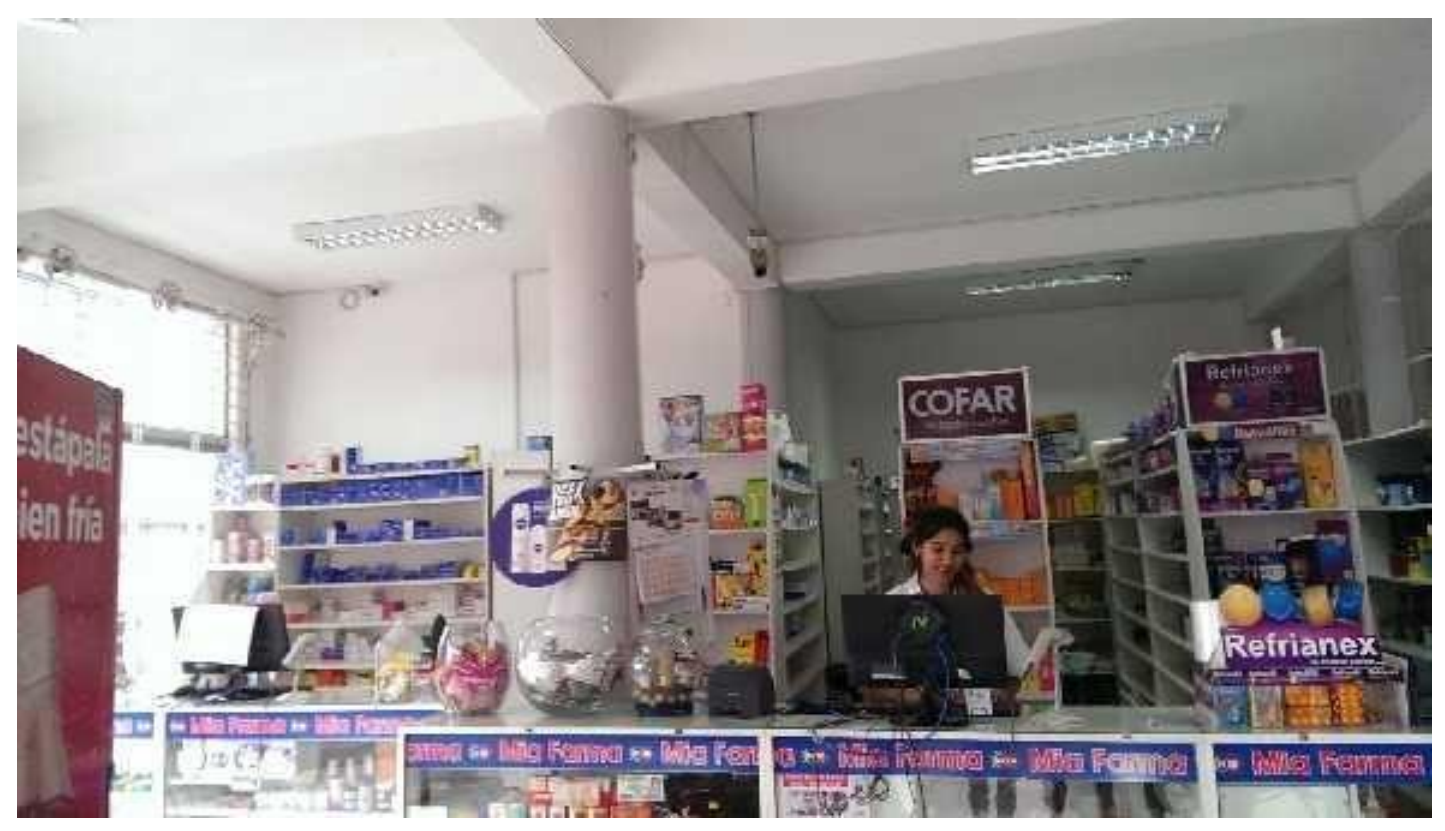

Figura 8. Fotografías de los negocios donde utilizo la aplicación № 2

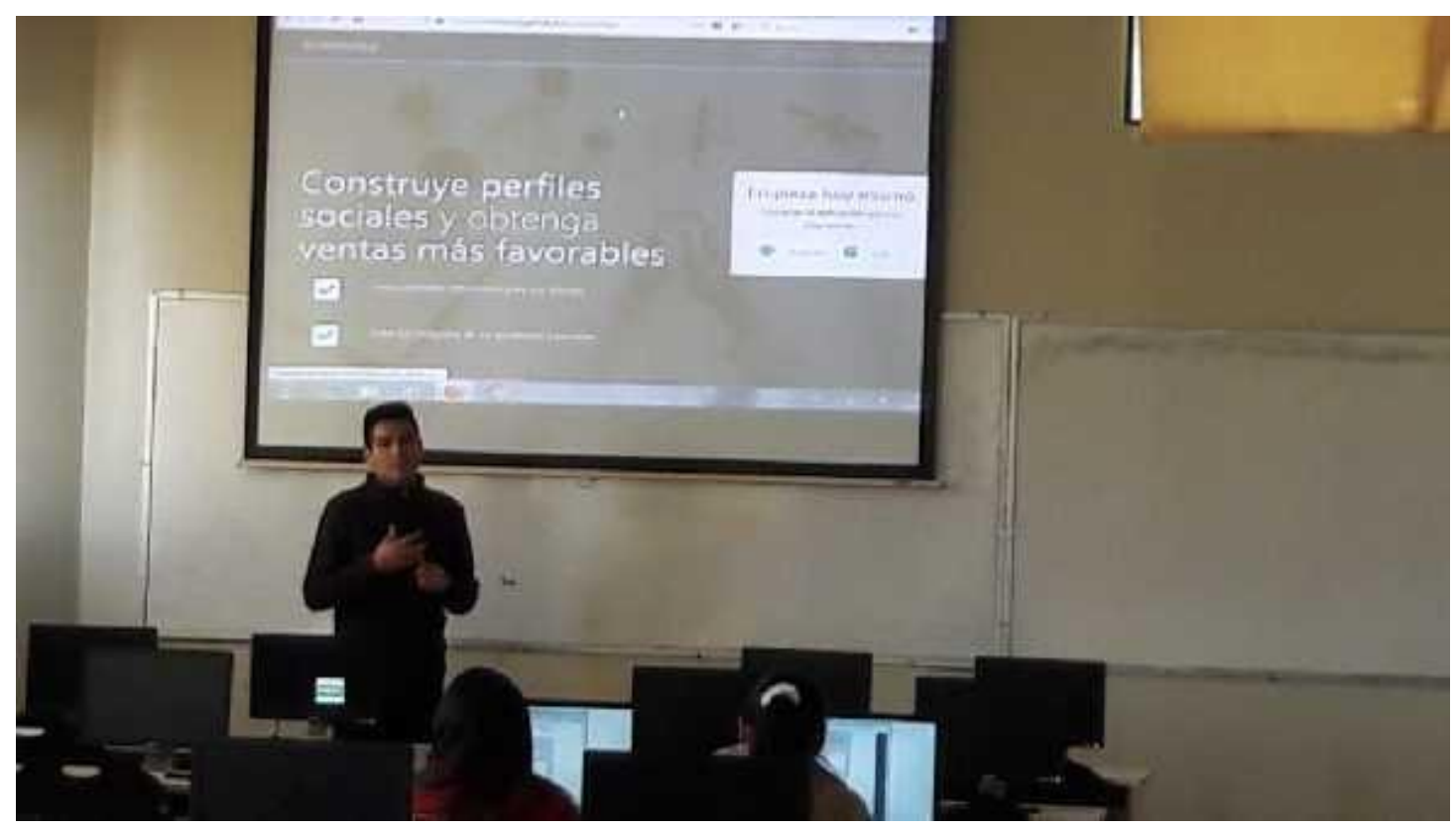

Figura 9. Explicación sobre las diapositivas de la aplicación № 3 


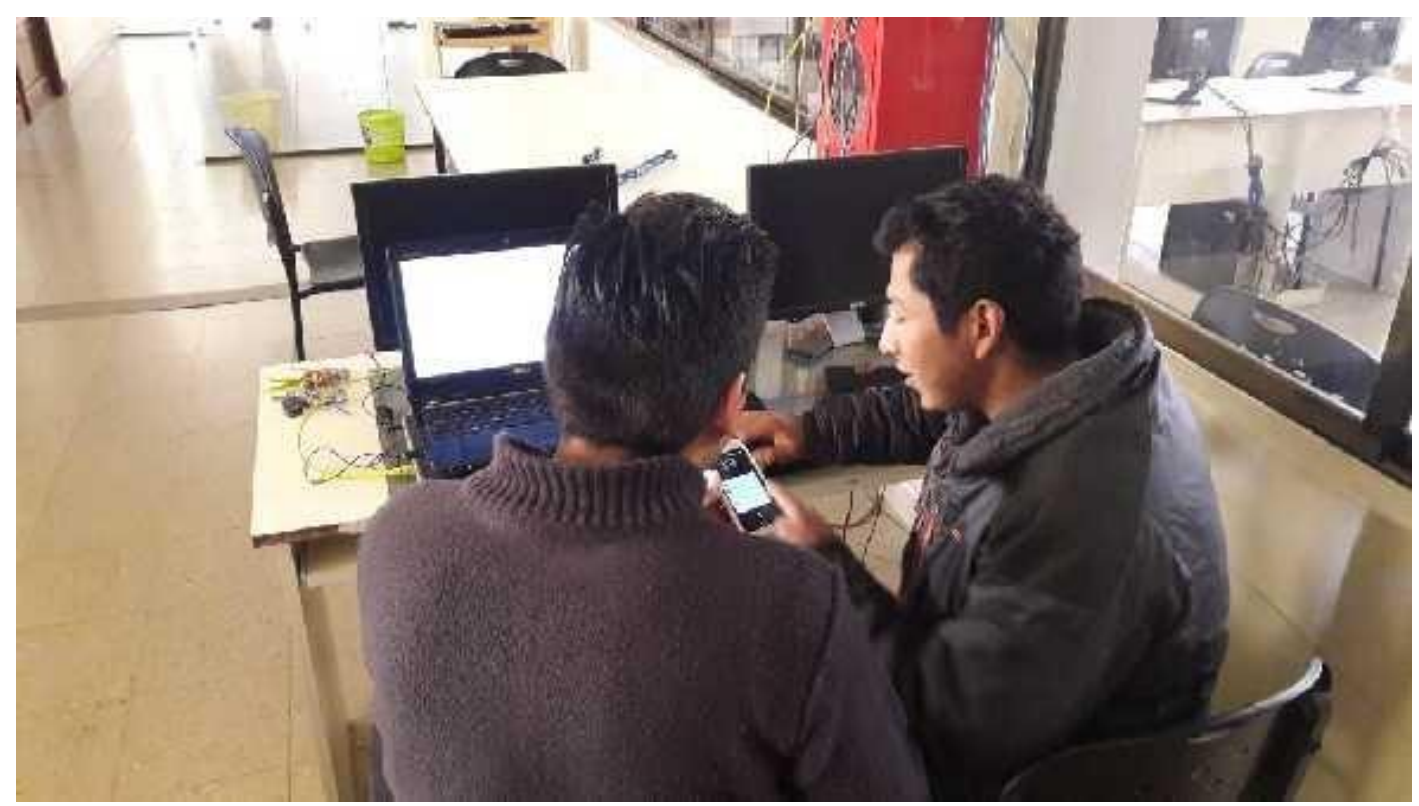

Figura 10. Orientación de manejo y uso de la aplicación № 4

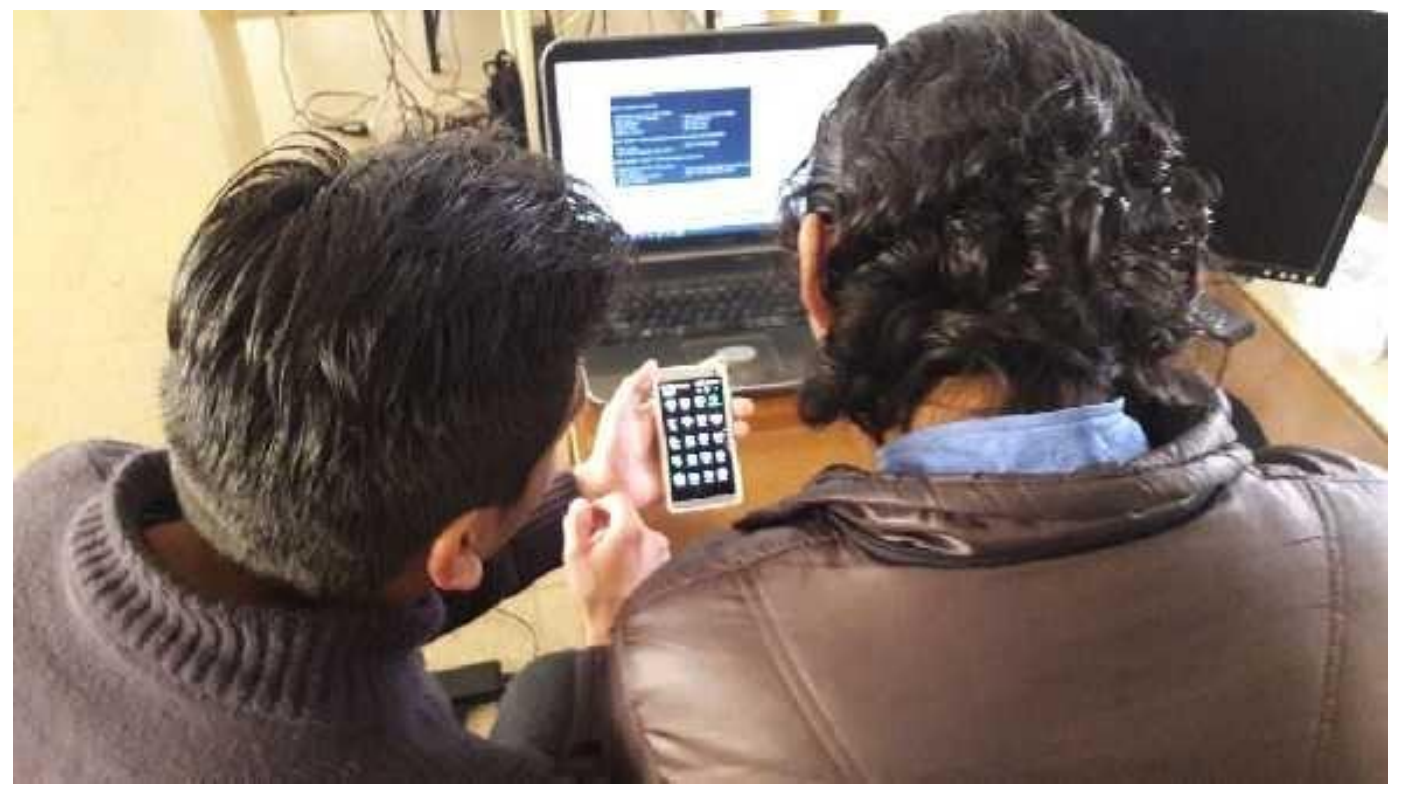

Figura 11. Orientación de manejo y uso de la aplicación № 5 


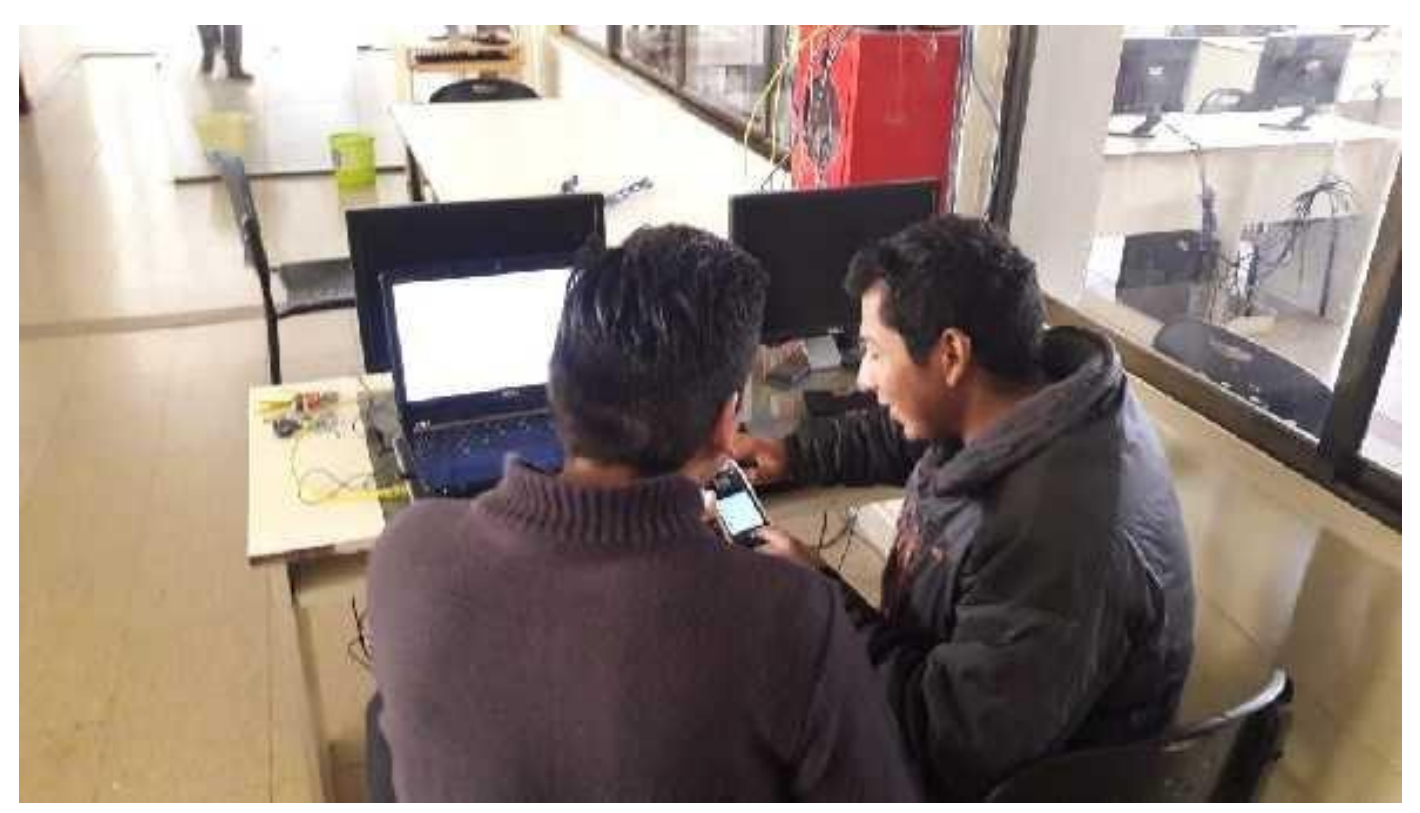

Figura 12. Orientación de manejo y uso de la aplicación №6

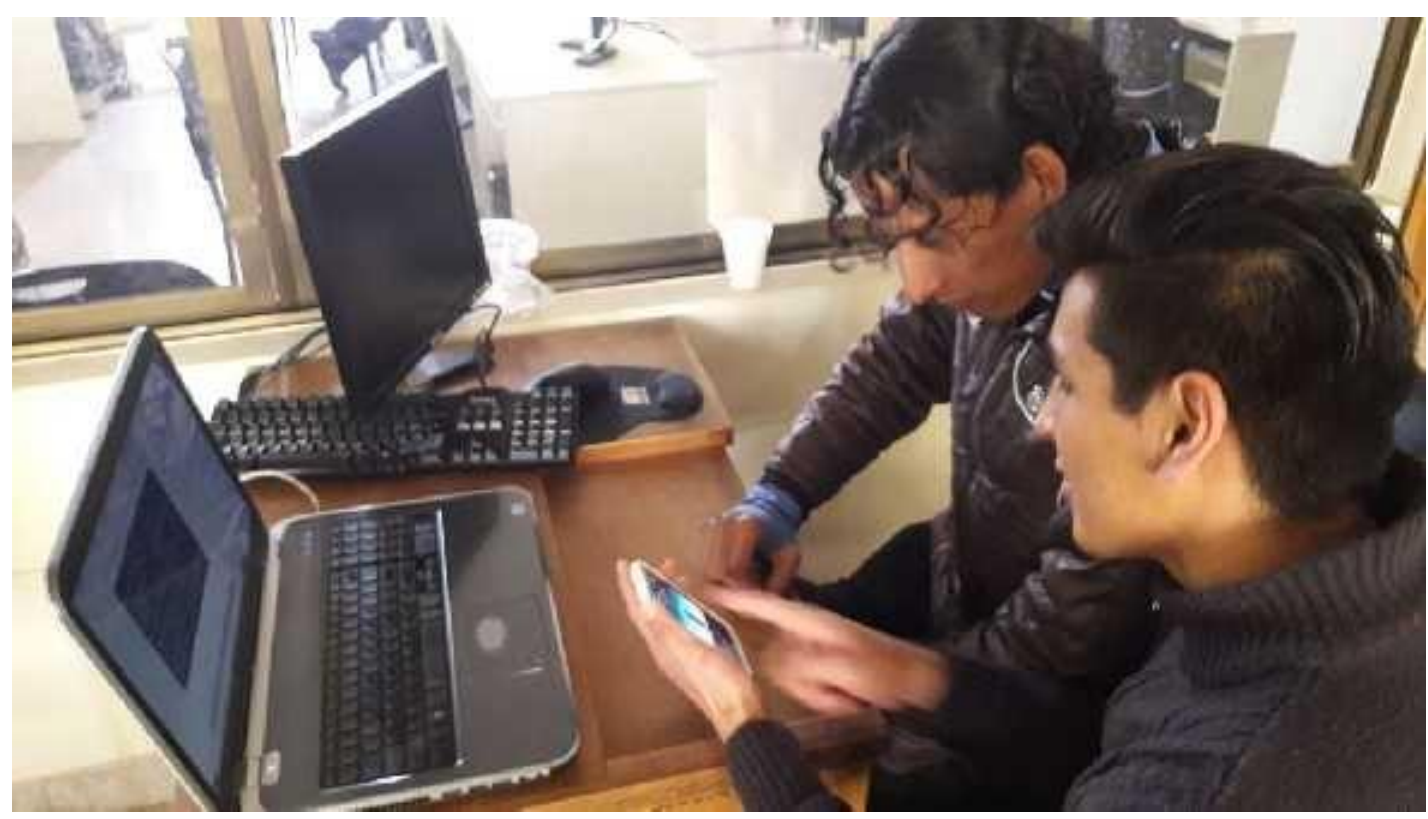

Figura 13. Orientación de manejo y uso de la aplicación № 7 


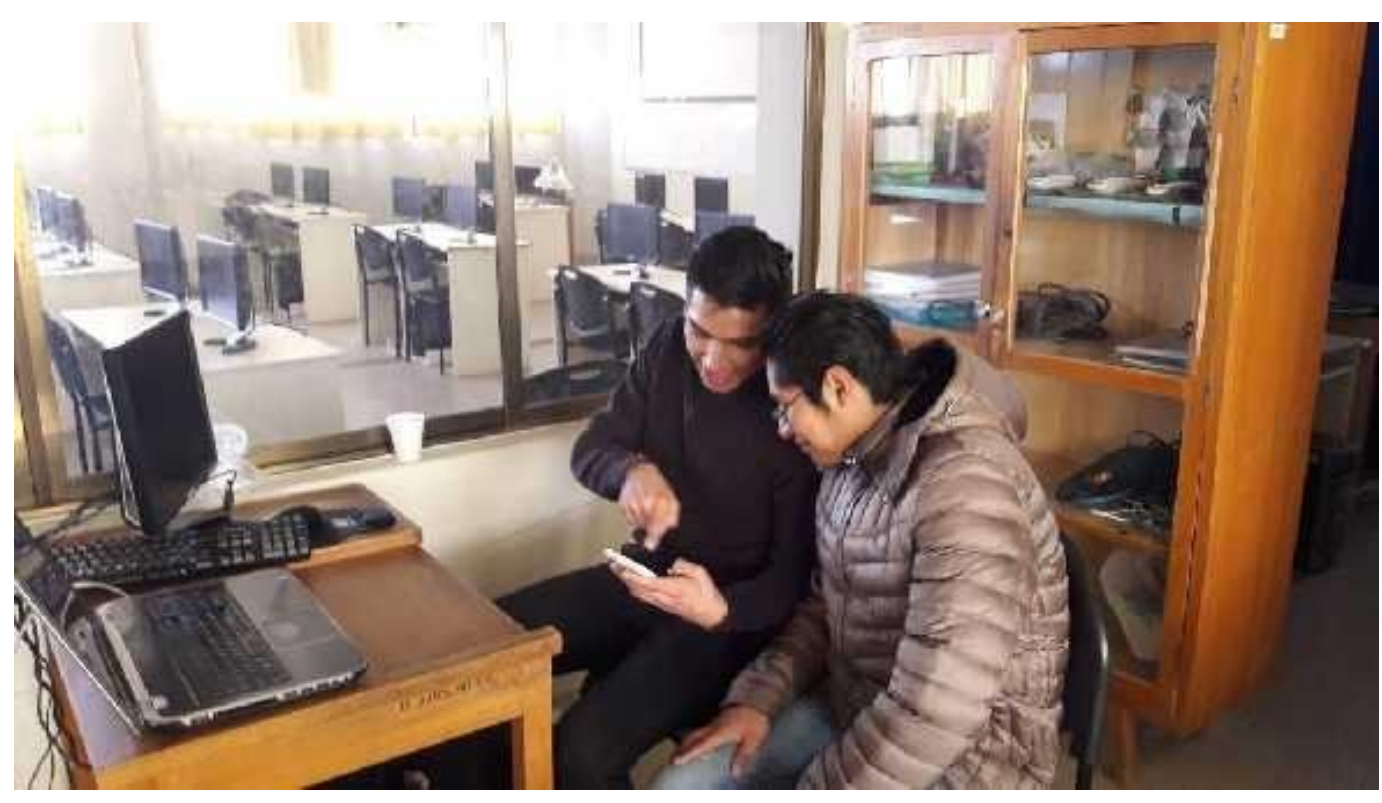

Figura 14. Orientación de manejo y uso de la aplicación №8

\section{Realización de Informe de Capacitación}

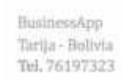

\section{INFORME DE ESTADO CAPACITACIÓN}

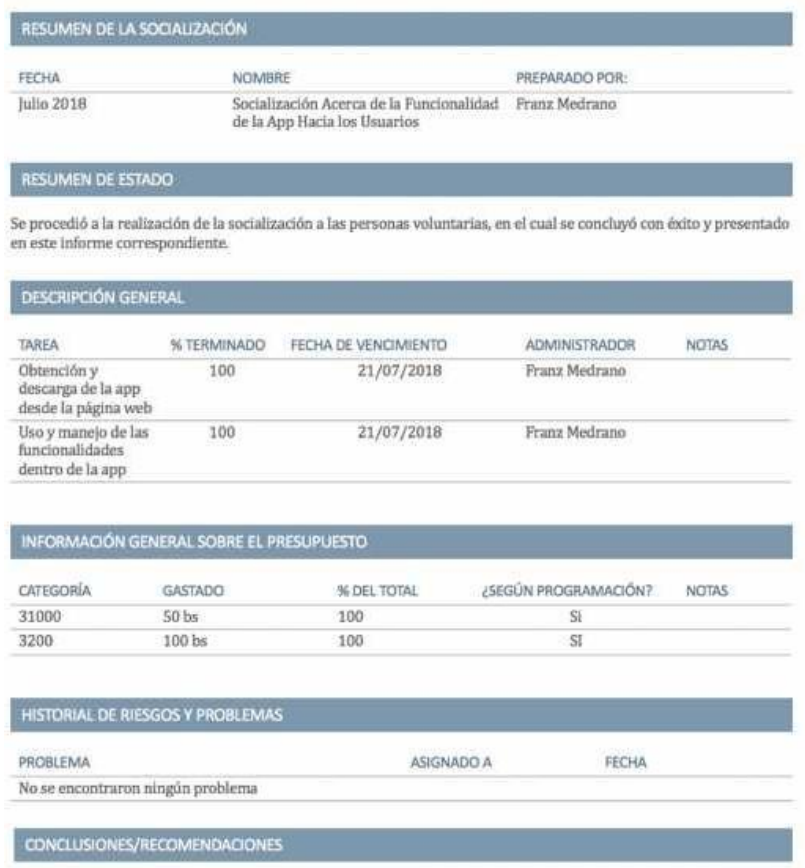

Se concibe en esta etapa como satistactoria y exitosa por parte de los usuarios en la colataoración y mancịo asi también en los exponentes realizando una adecuada y correcta presentación, ahora los usuarios ya se encuentran disponibles orientados para una correcta uttlización de nuestra aplicación "BusinessApp".

Figura 15. Informe de estado socialización 
CONCLUSIONES

Luego de haber culminado exitosamente con el desarrollo del proyecto, se llegó a las siguiente:

- Se espera que la aplicación móvil puesta en producción permita beneficiar tanto a los clientes como a los gerentes en la ciudad de Tarija, Bolivia, logrando una mejor comunicación informativa entre ambos.

- Se obtuvieron y generaron nuevos conocimientos en el manejo de la tecnología relacionada con las aplicaciones móviles, detrás de todo esto se pudo apreciar la valoración de los aprendizaje obtenido mediantes el desarrollo de las contextualizaciones, conceptos importantes en la metodología de desarrollo en software de los dispositivos móviles.

- A la vez se consiguió trabajar y utilizar diferentes API's que hacen posible la facilitación de resolución de problemas al trabajar con diferentes dispositivos.

- Se dictaron diferentes cursos de diseño y programación para llegar a implantar a la aplicación, cumpliendo con una experiencia enriquecedora $\mathrm{y}$ de fácil uso $\mathrm{y}$ acceso.

- A medida que la aplicación avanzaba fue tomando forma, se realizaron los correctivos correspondientes mediante cambios en la programación para resolver problemas inmediatos siguiendo siempre a secuencia lógica de los sistemas informáticos con relación a las labores que se realizan las personas mediante la realidad del día a día.

\section{Algunas sugerencias}

Las recomendaciones son necesarias para mantener la aplicación de acuerdo a sus especificaciones de calidad y uso, basado en los siguientes puntos:

- Los errores inoportunos surgidos pueden ser resueltos con una actualización de software, bajo una nueva versión y subiendo nuevamente a la página web.

- Se recomienda realizar backups de la base de datos del servidor cada 6 meses, y una revisión del tráfico de datos que envían los usuarios.

- Es recomendable revisar el tráfico de google maps platform con sus respectivas credenciales ya que este tiene un tiempo de caducidad, una serie de peticiones permitidas durante un mes.

- Los administradores deben estar constantemente monitoreando las actividades de los usuarios dentro de la aplicación.

\section{Que se espera}

- Con el paso del tiempo esperamos seguir implementando funciones adicionales con diferentes servicios de Firebase, la cual esperamos poder seguir implementar y ofrecer muchas mejoras a la aplicación.

- Crear diferentes módulos integrados para lograr un mayor alcance de nuevos usuarios en distintas áreas, beneficiando hacia los mismos.

- Se pretende utilizar de más proveedores de autenticación como Facebook, GitHub, Twitter en el registro de usuarios.

- Además, se propone utilizar animaciones de imágenes en nuestra capa de front-end, ofreciendo una ayuda adicional a la hora de utilizar la aplicación. 
- Rediseñar y ampliar las pantallas a la hora de utilizar según el nuevo mercado de dispositivos móviles Android y iOS conforme aparezcan.

- Se implementarán muchos más atributos para el registro de productos y servicios, para obtener un resultado especifico cuando se realicen las búsquedas con filtro de los mismos.

\section{Enlaces (Url's)}

\section{BIBLIOGRAFÍA}

URL de la App:

https://franzamd.github.io/BusinessA $\mathrm{pp} /$

URL de la App:

https://goo.gl/forms/uXiKy4JTFDkpQ wSI2

URL de la App:

https://goo.glforms/Z6CyW4CoV5GJk $79 \mathrm{e} 2$

Base, N. (2018). Docs Native Base. Obtenido de Introduction: https://docs.nativebase.io Base, N. (s.f.). Nativebase. Obtenido de Introduction:

https://docs.nativebase.io

Elixir. (s.f.). noticias es. Obtenido de elixir: http://e-lixir.es/que-es-unaappnativa/

Firebase. (s.f.). Firebase Google. Obtenido de Firebase: https://firebase. google.com

Francis, P. (2017). Mobile App Development Process. Obtenido de
THE BHW GROUP: https://thebhwgroup.com/blog/mob ile-app-development-process

ICESI, U. (2009). Modelos de negocio en Internet. Obtenido de Modelos de negocio en Internet: https://www.icesi.edu.co/blogs_estu diantes/vanessa/2009/09/17/model os-de- negocio-en-internet-b2bb2cc2cb2g/international, e. (June de 2015). ECMAScript 2015 Language Specification. Obtenido de ecma international: http://www.ecmainternational.org/ecma262/6.0/index.html\#sec-scope

Navigation, R. (2018). React Navigation. Obtenido de Getting started: https://reactnavigation.org/docs/en /getting-started.html

ReactJS. (s.f.). react. Obtenido de React: https://reactjs.org

React-Native. (2018). Facebook. Obtenido de Facebook React-Native: https://facebook.github.io/reactnative/

Vonovak. (s.f.). Github. Obtenido de reactnative-fetch-blob:

https://github.com/vonovak/reactnative-fetch-blob

Yeeply. (24 de noviembre de 2015). UX UI $I x D$. Obtenido de Diseño de apps móviles:

¿Qué es UX, UI y IxD?: https://www.yeeply.com/blog/disen o-de-apps-moviles-ux- ui-ixd/ 